\title{
Pterygium concomitant with other ocular surface lesions: Clinical implications and pathogenetic links
}

\author{
EFSTATHIOS T. DETORAKIS ${ }^{1}$, GEORGE KYMIONIS ${ }^{2}$, MICHAEL TSATSOS $^{3}$ and DEMETRIOS A. SPANDIDOS ${ }^{4}$ \\ ${ }^{1}$ Department of Ophthalmology, University Hospital of Heraklion, Heraklion 71110; ${ }^{2}$ Institute of Vision and Optics, \\ University of Crete, Heraklion 71003, Greece; ${ }^{3}$ Moorfields Eye Hospital, London EC1V 2PD, UK; \\ ${ }^{4}$ Laboratory of Clinical Virology, University of Crete, Medical School, Heraklion 71409, Greece
}

Received October 12, 2015; Accepted November 13, 2015

DOI: $10.3892 /$ etm.2015.2865

\begin{abstract}
In the present study, we aimed to evaluate the co-existence of ophthalmic pterygium with other ocular surface lesions in a cohort of patients from the Cretan population. This is a retrospective evaluation of all pterygia in patients examined and treated at the Department of Ophthalmology of the University Hospital of Heraklion, Greece during an 8-year period (from June 2006 to June 2014). A total of 158 cases was examined. Ocular surface images and medical history were evaluated in order to detect concomitant ocular surface pathological conditions. Concomitant lesions included conjunctival nevi (5 cases, $3.16 \%$ ), iris nevi (4 cases, $2.53 \%$ ), conjunctival papillomas (8 cases, $5.06 \%$ ), conjunctival intraepithelial neoplasia (CIN; 4 cases, $2.53 \%$ ) and 6 cases of hypertophy of the plica semilunaris $(3.79 \%)$. Of note, pterygium was overlying the iris which was occupied by the iris nevus in 2 out of the 4 cases of iris nevus. Overall, our data indicate that ophthalmic pterygium may often co-exist with other clinically significant ocular surface lesions. The association of ophthalmic pterygium with conjunctival papillomas or CIN stresses the potential involvement of human papilloma virus in the pathogenesis of ophthalmic pterygium, whereas the topographical association of pterygium with iris nevus may offer support to the transcameral light pathway pathogenetic mechanism.
\end{abstract}

\section{Introduction}

Pterygium is a common ocular surface condition which may expand on the cornea and thus threaten vision through various mechanisms, such as visual axis occupation or the induction of significant astigmatism (1). A strong association between ophthalmic pterygium and exposure to solar radiation, particu-

Correspondence to: Dr Efstathios T. Detorakis, Department of Ophthalmology, University Hospital of Heraklion, Heraklion 71110, Greece

E-mail: detorakis@hotmail.com

Key words: ophthalmic pterygium, conjunctival nevus, conjunctival papillomas, conjunctival intraepithelial neoplasia, hypertrophy of the plica semilunaris larly to the ultraviolet (UV) light from the solar spectrum, has been reported $(1,2)$. Moreover, a number of previous studies have provided evidence of oncogenic activity in cell populations obtained from pterygium $(1,3-5)$, possibly originating from corneo-scleral stem cells $(3,4)$, whereas the detection of human papilloma virus (HPV) DNA in ophthalmic pterygia has raised the possibility of viral participation in the pathogenesis of pterygium (6-9). The considerable diversity in the mechanisms associated with the development of pterygium suggests that the same mechanisms may also be involved in the development of other common ocular surface conditions. In addition, significant geographical- and population-related differences in the incidence of pterygium have been reported, possibly reflecting genetic and environmental pathogenetic effects $(9,10)$.

Crete is a region with interesting epidemiological and demographical features associated with pterygium, such as increased exposure to solar light and a marked diversity in altitudes of residence (also associated with differences in the exposure to UV radiation) (5). Previous studies on pterygia in patients originating from the Cretan population have detected molecular genetic alterations, such as the loss of heterozygosity and microsatellite instability, as well as the involvement of HPV, suggesting a destabilized genetic background for ocular surface cell populations in affected eyes $(1,4,5)$. In the present study, we aimed to determine whether the presence of ophthalmic pterygium may be associated with an increased likelihood for the development or presence of other ocular surface lesions in the same patients from the Cretan population.

\section{Patients and methods}

This study analyzed, in a retrospective manner, a cohort of patients with ophthalmic pterygium treated at the Department of Ophthalmology of the University Hospital of Heraklion (Heraklion, Greece) for 8 consecutive years (2006-2014). The medical history of the patients was examined and clinical data were recorded, including patient age and gender, the age at which pterygium was diagnosed for the first time, the surgical history of pterygium (whether it was primary or recurrent), the location of pterygium on the ocular surface (nasal, temporal or both) and the size of pterygium (in mm of advancement on the corneal surface). Clinical ocular surface images were also 


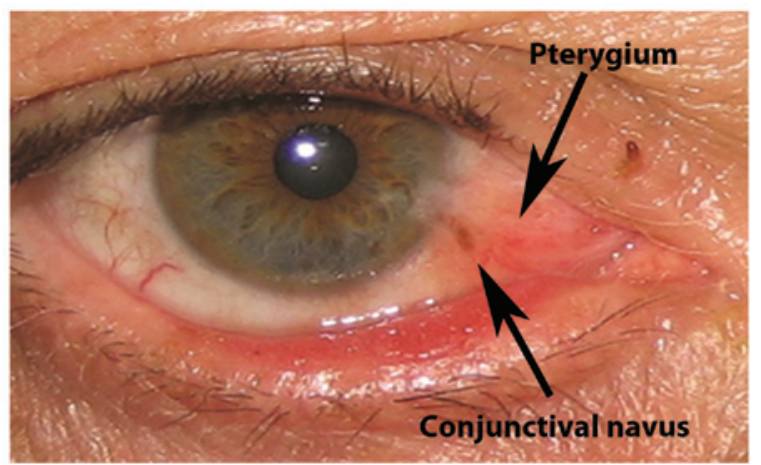

Figure 1. A case of conjunctival nevus located on the body of pterygium (both indicated by black arrows).

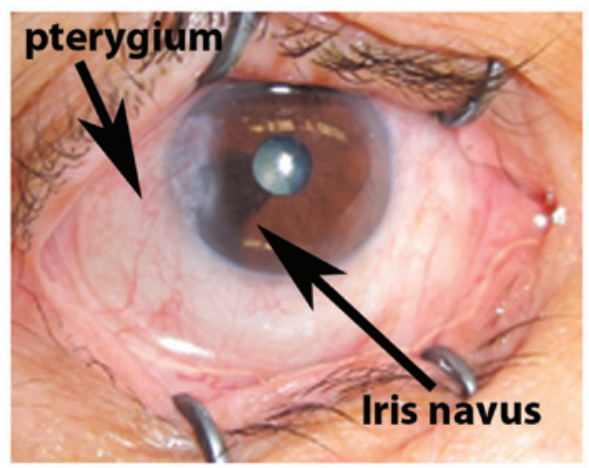

Figure 2. A case of temporal pterygium and impsilateral iris nevus. The iris nevus occupies the same sector of the iris covered by the temporal pterygium (both indicated by black arrows).

examined in order to assess the degree of pterygium vascularity (on a scale of 1-4). The concomitant detection of any other ocular surface lesions [which were present in the eye(s) of the same patient at the time of the initial clinical examination] was recorded. All chart evaluations and clinical image assessment were performed by the same experienced ocular surface surgeon (E.T.D.).

The findings were statistically analyzed using the statistical package SPSS 8.0 (SPSS, Inc., Chicago, IL, USA). The incidence of each concomitant condition within the population of patients with ophthalmic pterygium was recorded. Correlations between the demographic and clinical characteristics of the patients in our cohort (such as the age of the patients, age at which the first occurrence of pterygium was reported, the size and vascularity of pterygium, as well as a history of recurrence) and the co-existence of pterygium with other ocular surface lesions were examined using Pearson's bivariate correlation coefficient. Statistical significance was set at a value of $\mathrm{p}<0.05$.

\section{Results}

Overall, 158 cases of pterygium (96 males, 60.75\%) were included in this study. The age of the patients on their first examination [mean \pm standard deviation (SD), range] was $67.23 \pm 12.14(45-84)$ years in the male patients in our cohort and $71.38 \pm 10.112(48-86)$ years in the female patients. The mean age of the patients when the pterygium first appeared was

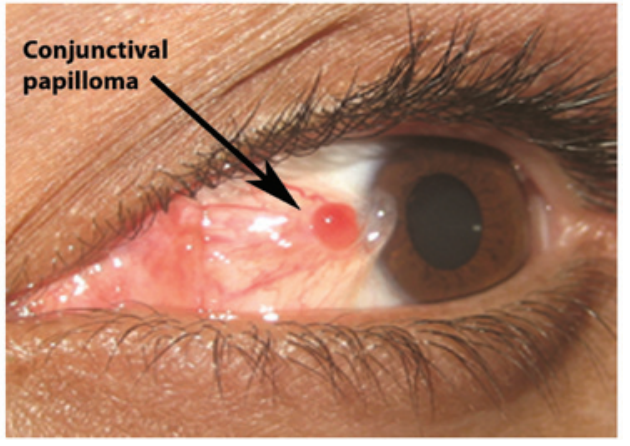

Figure 3. A case of conjunctival papilloma on the body of a pterygium (indicated by black arrow).

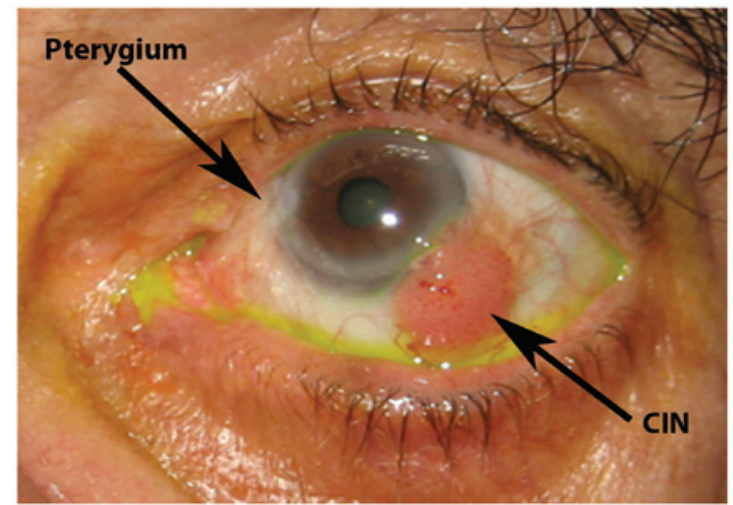

Figure 4. A case of conjunctival intraepithelial neoplasia (CIN) on the temporal limbus concomitant with pterygium on the nasal limbus (both indicated by black arrows).

$37.89 \pm 16.24(25-79)$ years in the male patients in our cohort and $42.51 \pm 18.08$ (28-80) years in the female patients. Pterygium was located nasally in 140 cases $(88.60 \%)$, temporally in 5 cases $(3.67 \%)$ and on both the nasal and temporal sides in 13 cases $(8.22 \%)$ in the affected eyes. On the first examination at the Department of Ophthalmology of the University Hospital of Heraklion, pterygium was primary in 114 cases $(73.54 \%)$. The mean pterygium vascularity was $3.11 \pm 2.9$ (1-4) and $2.98 \pm 2.56(1-4)$ in the male and female patients, respectively. The mean protrusion of pterygium on the corneal surface was $3.68 \pm 1.99(1-5) \mathrm{mm}$ and $3.55 \pm 2.24(1-4) \mathrm{mm}$ in the male and female patients, respectively.

Ocular surface lesions which were recorded to co-exist with pterygium included conjunctival nevi (5 cases, 3.16\%), iris nevi (4 cases, 2.53\%), biopsy-proven conjunctival papillomas ( 8 cases, $5.06 \%$ ), biopsy-proven conjunctival intraepithelial neoplasia (CIN; 4 cases, $2.53 \%$ ) and hypertophy of the plica semilunaris (6 cases, 3.79\%). Representative cases of pterygium with concomitant conjunctival nevus, iris nevus, conjunctival papillomas, biopsy-proven CIN and hypertophy of the plica semilunaris are presented in Figs. 1-5, respectively. The correlation between the clinical and demographical parameters examined (including the patient age, recurrence history, the age at which pterygium first appeared or pterygium vascularity) and the presence of concomitant ocular surface lesions in the patients with pterygium was not statistically significant in all cases. The values of Pearson's bivariate correlation coefficient 
Table I. Pearson's bivariate correlation coefficient values for the correlations between the clinical and demographic parameters of the patients with pterygium and concomitant other ocular surface lesions, as well as respective p-values.

\begin{tabular}{lcc}
\hline Parameter & r-value & p-value \\
\hline $\begin{array}{l}\text { Patient age } \\
\text { Male }(67.23 \pm 12.14,45-84 \text { years })\end{array}$ & -0.09 & 0.78 \\
$\quad \begin{array}{l}\text { Female }(71.38 \pm 10.112,48-86 \text { years }) \\
\text { Age at which pterygium first appeared }\end{array}$ & -0.19 & 0.37 \\
$\quad \begin{array}{l}\text { Male }(25-79 \text { years }) \\
\text { Female }(28-80 \text { years })\end{array}$ & & \\
$\begin{array}{l}\text { Pterygium vascularity } \\
\text { Male }(3.11 \pm 2.9 ; 1-4)\end{array}$ & 0.24 & 0.31 \\
$\quad \begin{array}{l}\text { Female }(2.98 \pm 2.56 ; 1-4) \\
\text { Pterygium size } \\
\quad \text { Male }(3.68 \pm 1.99 ; 1-5) \mathrm{mm}\end{array}$ & 0.27 & 0.22 \\
$\quad$ Female $(3.55 \pm 2.24 ; 1-4) \mathrm{mm}$ & & \\
Recurrence history & 0.25 & 0.29 \\
\hline
\end{tabular}

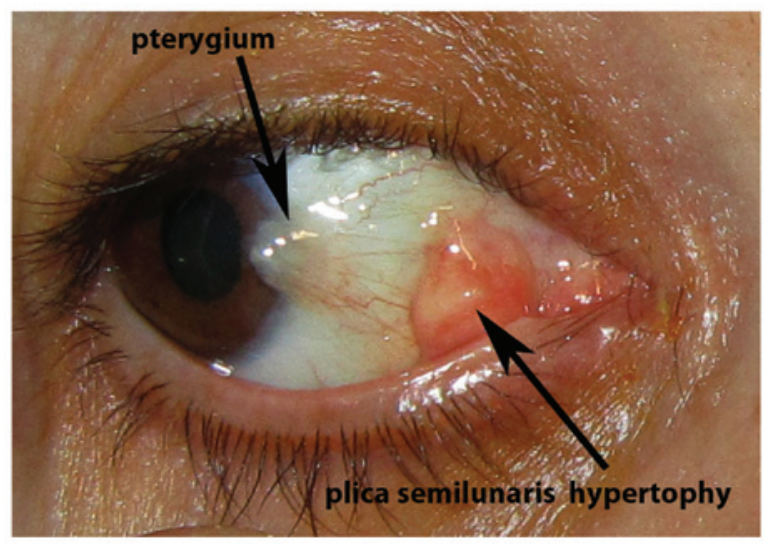

Figure 5. A case of hypertophy of the plica semilunaris associated with pterygium (both indicated by black arrows).

for the correlations between the parameters examined and the presence of concomitant ocular surface lesions, as well as the respective p-values are presented in Table I. In the case of iris nevus, 2 cases were reported in which the nevus corresponded topographically with pterygium, i.e., the iris nevi were located on the iris sector corresponding to the corneal surface occupied by pterygium (Fig. 5). In these cases, iris nevi were reported to be congenital (in 1 case) or to develop long before to the appearnce of pterygium (in 1 case).

\section{Discussion}

In this study, we evaluated the likelihood of the co-existence between ophthalmic pterygium and other ocular surface lesions in the Cretan population. The results suggested that pterygium may co-exist with several other ocular surface conditions and that pathogenetic links may exist between pterygium and other concomitant lesions, such as iris nevi.

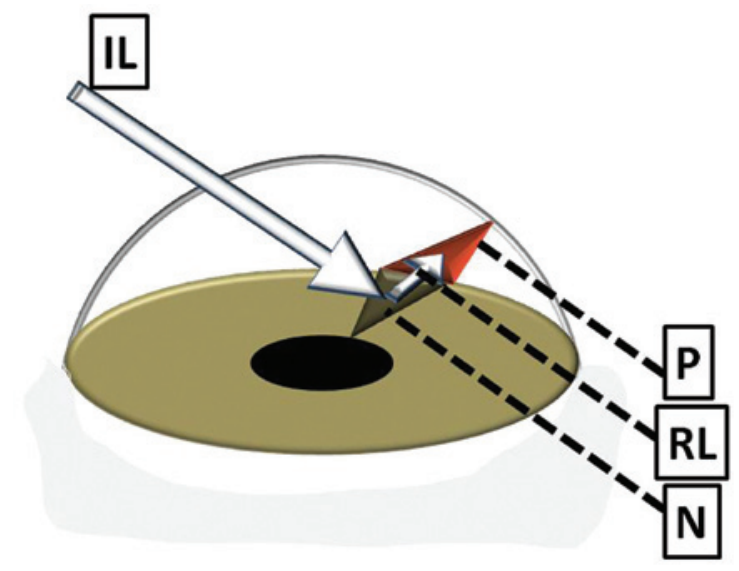

Figure 6. Diagrammatic presentation of the potential light reflection from the iris nevus surface to the inner corneal surface, as a potential pathogenetic mechanism for the development of pterygium. IL, incident light; RL, reflected light; N, nevus; P, pterygium.

It has long been known that pterygium may display unexpected histological characteristics, compatible with neoplastic lesions (11). Moreover, previous studies have examined the prevalence of various ocular surface lesions in patients with pterygium $(12,13)$. CIN has been reported in $1.89 \%$ of patients with pterygium (11), whereas ocular surface melanocytic lesions have been found in $11.1 \%$ of patients with pterygium (13). The respective prevalences from the present study are in agreement with these reports. The potential co-existence of such ocular surface lesions with pterygium suggests that the clinician managing pterygium cases should be prepared to detect and adequately address additional concomitant ocular surface pathologies. These findings also suggest that pterygium and other concomitant ocular surface conditions may share common pathogenetic pathways, such as the effects of UV radiation or the activity of HPV.

The association of pterygium with hypertophy of the plica semilunaris has previously been mentioned (14) and suggests that inflammatory activity in the area of the plica semilunaris may play a key role in the pathogenesis of some pterygia. The association of pterygium with conjunctival papillomas may reflect the presence of HPV on the ocular surface, as previously mentioned $(1,6,7)$. In the case of iris nevi, the prevalence detected in the eyes of patients in our study cohort was similar with that reported for iris melanocytic lesions in the general Caucasian population (4-6\%) (15), reflecting the genetic Caucasian profile of the Cretan population. It should be noted however, that iris nevi were topographically associated with pterygium in this study in 2 out of 4 cases recorded, displaying an association between the borders of the iris nevus and the borders of the overlying pterygium. One potential explanation for this finding may be found in the concept of transcameral light pathways proposed by Coroneo as a pathogenetic mechanism for the development of pterygium (concept of peripheral light focusing) (16). In this proposed model, light scattered in the anterior chamber may deviate from the transpupillary course and be directed towards the limbus. The ab interno irradiation of limbal stem cells may cause genetic destabilization, eventually leading to 
the development of pterygium. Accordingly, it can be hypothesized that alterations in the reflectivity of the anterior iris surface due to the presence of an iris nevus may enhance the effect of transcameral light pathways on the iris sector corresponding to the nevus, and may thus increase the possibility of the development of ipsilateral pterygium (Fig. 6).

The retrospective design may be considered a weakness of the present study. Moreover, the overall number of cases recorded may be considered low and this may possibly explain the lack of statistically significant correlations between the recorded clinical and demographic parameters of thee patients in our cohort and the presence of concomitant pathological conditions. On the other hand, the fact that all pterygia consecutively managed at the same reference centre during an 8-year period were included, whereas the genetic background of the patients studied was homogeneous (originating from the Cretan population) may enhance the validity of our results.

In conclusion the findings of the present study suggest that potential pathogenetic links may exist between pterygium and other clinically significant ocular surface pathological conditions. The hypothesis that transcameral light pathways are associated with iris lesions needs to be further evaluated in larger multicenter studies, recruiting larger numbers of cases.

\section{References}

1. Detorakis ET and Spandidos DA: Pathogenetic mechanisms and treatment options for ophthalmic pterygium: Trends and perspectives (Review). Int J Mol Med 23: 439-447, 2009.

2. Coroneo MT: Pterygium as an early indicator of ultraviolet insolation: a hypothesis. Br J Ophthalmol 77: 734-739, 1993.

3. Chui J, Coroneo MT, Tat LT, Crouch R, Wakefield D and Di Girolamo N: Ophthalmic pterygium: a stem cell disorder with premalignant features. Am J Pathol 178: 817-827, 2011.
4. Detorakis ET, Zaravinos A and Spandidos DA: Growth factor expression in ophthalmic pterygia and normal conjunctiva. Int $\mathbf{J}$ Mol Med 25: 513-516, 2010

5. Detorakis ET, Sourvinos G, Tsamparlakis J and Spandidos DA: Evaluation of loss of heterozygosity and microsatellite instability in human pterygium: clinical correlations. Br J Ophthalmol 82: 1324-1328, 1998

6. Detorakis ET, Drakonaki EE and Spandidos DA: Molecular genetic alterations and viral presence in ophthalmic pterygium. Int J Mol Med 6: 35-41, 2000.

7. Detorakis ET, Sourvinos G and Spandidos DA: Detection of herpes simplex virus and human papilloma virus in ophthalmic pterygium. Cornea 20: 164-167, 2001.

8. Gallagher MJ, Giannoudis A, Herrington CS and Hiscott P: Human papillomavirus in pterygium. Br J Ophthalmol 85: 782-784, 2001

9. Piras F, Moore PS, Ugalde J, Perra MT, Scarpa A and Sirigu P: Detection of human papillomavirus DNA in pterygia from different geographical regions. Br J Ophthalmol 87: 864-866, 2003.

10. Saw SM and Tan D: Pterygium: prevalence, demography and risk factors. Ophthalmic Epidemiol 6: 219-228, 1999.

11. Degrassi M, Piantanida A and Nucci P: Unexpected histological findings in pterygium. Optom Vis Sci 70: 1058-1060, 1993.

12. Artornsombudh P, Sanpavat A, Tinnungwattana U, Tongkhomsai V, Sansopha L and Tulvatana W: Prevalence and clinicopathologic findings of conjunctival epithelial neoplasia in pterygia. Ophthalmology 120: 1337-1340, 2013.

13. Perra MT, Colombari R, Maxia C, Zucca I, Piras F, Corbu A, Bravo S, Scarpa A and Sirigu P: Finding of conjunctival melanocytic pigmented lesions within pterygium. Histopathology 48: 387-393, 2006.

14. Detorakis ET, Halkia A, Tsakalaki V and Spandidos DA: Association between pterygium and plica semilunaris morphology. Clin Experiment Ophthalmol 41: 891-892, 2013.

15. Harbour JW, Brantley MA Jr, Hollingsworth H and Gordon M: Association between posterior uveal melanoma and iris freckles, iris naevi, and choroidal naevi. Br J Ophthalmol 88: 36-38, 2004.

16. Coroneo M: Ultraviolet radiation and the anterior eye. Eye Contact Lens 37: 214-224, 2011. 